\title{
ОПТИМИЗАЦИЯ ГЕОМЕТРИИ ЗУБЦОВОЙ ЗОНЫ ГИСТЕРЕЗИСНОЙ МУФТЫ ЗАПОРНОЙ АРМАТУРЫ НЕФТЕПРОВОДА
}

\author{
Гарганеев Александр Георгиевич',
} garganeev@rambler.ru

Динь Конг Кюи', caothuhanam89@gmail.com

\section{Сипайлова Надежда Юрьевна', snu2002@mail.ru}

\author{
Кашин Евгений Игоревич', \\ kashinei@sibmail.com \\ 1 Национальный исследовательский Томский политехнический университет, \\ Россия, 634050, г. Томск, пр. Ленина, 30.
}

\begin{abstract}
Актуальность работы связана с поиском новых эффективных технических решений в области электроприводов запорной арматуры трубопроводного транспорта нефтепродуктов на основе использования гистерезисного принципа электромеханического преобразования энергии и новых магнитотвердых материалов.

Цель: выявить оптимальную геометрию зубцовой зоны электромагнитной гистерезисной муфты на базе сплава Fe-Cr-Co для получения максимального вращающего момента на основе анализа процессов перемагничивания гистерезисного слоя.

Методы: экспериментальные методы исследования магнитных характеристик магнитотвердых материалов с использованием оригинальных лабораторных установок; имитационное моделирование в программе ANSYS MAXWELL; теоретические методы анализа электромагнитного поля.

Результаты. Для гистерезисных муфт на базе сплава Fe-Cr-Co 22X15KА получены и проанализированы зависимости вращающего момента от геометрических параметров зубцовой зоны муфты. Для оценки эффективности использования гистерезисного материала авторами введен коэффициент использования гистерезисного слоя по тангенциальной составляющей магнитного поля. В программе ANSYS MAXWELL разработана имитационная модель электромагнитной гистерезисной муфты с неподвижной обмоткой управления, учитывающая параметры сплава Fe-Cr-Co 22X15KA. С помощью разработанной модели получены оптимальные значения геометрических размеров зубцовой зоны с целью достижения максимального вращающего момента при фиксированных габаритах и весе. Сделан вывод о том, что вариант электропривода трубопроводной запорной арматуры с гистерезисной муфтой и асинхронным электродвигателем благодаря простоте его реализации, возможности регулирования и ограничения момента в широком диапазоне температур и окружных скоростей можно рассматривать как перспективную альтернативу электроприводам с частотным управлением и тиристорными регуляторами.
\end{abstract}

\section{Ключевые слова:}

Электромагнитная муфта, моделирование, электропривод, момент, гистерезис, манитотвердый материал, запорная арматура, нефтепровод.

\section{Введение}

В многочисленных электроприводных системах электрический двигатель соединяется с производственным механизмом через муфту, служащую для передачи механической энергии с ведущего вала на ведомый при ограничении крутящего момента. Весьма распространенными системами являются электроприводы запорной арматуры (ЭПЗА) для перекачки нефти и газа, в которых требование ограничения крутящего момента является обязательным условием безопасного и надежного функционирования трубопроводного транспорта нефтепродуктов [1-3]. ЭПЗА выполняет функции перекрытия сечения трубопровода с фиксированным ограничением момента приводного двигателя (ПД) в условиях широкого температурного диапазона и удаления от диспетчерских пунктов и центральных электросетей.

\section{Постановка задачи исследования}

Вопросы применения типа ПД и управляющего полупроводникового преобразователя (ПП) подробно рассмотрено в работе [4], в которой впервые предложено в ЭПЗА применение электрической машины гистерезисного типа, являющейся, по сути, электромагнитной муфтой. Одним из вариантов построения ЭПЗА на основе гистерезисного принципа преобразования энергии является ЭП на базе широко применяемого асинхронного двигателя (АД) и гистерезисной муфты (ГМ) с простым блоком для регулирования постоянного тока управления [5]. В этом случае электромагнитная гистерезисная муфта (ЭМГМ) располагается между АД и редуктором, предотвращая увеличение момента уплотнения клина задвижки сверх максимально допустимых значений. Если требуется изменить моменты уплотнения (вытяжки) применяется про- 
стейшая схема регулировки тока обмотки управления ЭМГМ на выходе или в составе выпрямителя В (рис. 1).

Гистерезисные муфты в режиме несинхронного вращения, торможения или пуска работают с постоянством момента, что может явиться решающим фактором для безотказной работы технологического механизма - запорной арматуры. При этом возможно устранение сложного блока электронного управления с частотными преобразователями или тиристорными регуляторами с ограничением вращающего момента на основе его идентификации, значительно снижающих надежность электропривода запорной арматуры $[1,6]$. В отличие от подобных систем, приводящих, фактически, к торможению, остановке приводного двигателя и, как следствие, к его нагреву, вариант с ЭМГМ предусматривает лишь останов ведомого вала муфты. Для реализации идеи применения ГМ в составе ЭПЗА необходимо провести ряд исследований, связанных с особенностями перемагничивания гистерезисного материала в составе ГМ, что позволит выявить оптимальную геометрию зубцовой зоны муфты с целью получения максимального вращающего момента при ее фиксированных габаритах и весе.

\section{Теория перемагничивания гистерезисного материала в конструкции муфты}

Конструктивные варианты гистерезисных магнитных и электромагнитных муфт рассмотрены в [5, 7-9]. Для применения в ЭПЗА представляет интерес конструкция ЭМГМ (рис. 1) с неподвижной внешней обмоткой, позволяющая обеспечить работу во взрывоопасной зоне при организации бесконтактного управления муфтой в непосредственной близости с нефте- или газопроводом. Следует отметить, что приведенную на рис. 1 , б немагнитную вставку (поз. 8) можно не использовать, изготовив внешний индуктор из одного ферромагнитного материала, поскольку сечение зубцовой зоны значительно превы- шает сечение элемента поз. 8. Обмотка управления 7 создает постоянный магнитный поток, регулировку которого при необходимости можно осуществлять изменением тока обмотки. В гистерезисном слое -4 за счет наличия зубцов внутреннего и внешнего индукторов образуется переменная составляющая магнитного потока, приводящая к возникновению потерь на гистерезис и постоянной величины гистерезисного момента, не зависящего от скорости вращения ведущего вала ЭМГМ. Зубцы внешнего и внутреннего индукторов ЭМГМ могут иметь различную геометрическую форму.

Традиционными магнитными материалами для гистерезисных двигателей и муфт следует считать сплавы типа 52КФ на основе $\mathrm{Fe}-\mathrm{Co}-\mathrm{V}$ (викаллой) c удельными потерями на гистерезис $p_{\mathrm{r}} \approx 0,05$ Дж/ $\mathrm{cm}^{3}$, а также сплавы типа $12 \Gamma \mathrm{H}$ на основе $\mathrm{Fe}-\mathrm{Ni}-\mathrm{Mn}$ и $12 \kappa \mathrm{B}$ на основе Fe-Co-W-Mo с $p_{\mathrm{r}} \approx 0,02$ Дж/ $\mathrm{cm}^{3}$. Данные сплавы обладают низкими удельными потерями на гистерезис и высокой стоимостью. Современным и перспективным материалом для гистерезисных муфт и двигателей является деформируемый сплав типа Fe-Cr-Co, обладающий высокими механическими и стабильными магнитными свойствами в широком температурном диапазоне и с большими для гистерезисных материалов значениями удельных потерь на гистерезис $\left(p_{\text {г }}=0,12 . . .0,18\right.$ Дж $\left./ \mathrm{cm}^{3}\right)$ и энергетического произведения $(B H)_{\max }=40 . .60 \mathrm{\kappa Дж} / \mathrm{m}^{3}$ $[10,11]$. Высокие прочностные свойства сплава Fe-Cr-Co способны конкурировать с высокопрочными сталями и титаном, что позволяет применять указанный сплав в безбандажных высокоскоростных механизмах. Согласно исследованиям работы [12], сплав типа 25X15K на основе Fe-Cr-Co при сохранении магнитных свойств имеет стабильные пластические и прочностные свойства до температуры $500{ }^{\circ} \mathrm{C}$. В различных структурных состояниях сплавы $\mathrm{Fe}-\mathrm{Cr}-\mathrm{Co}$ имеют предел прочности $\sigma_{\text {в }}=980 \ldots 1000 \mathrm{MПа}$, что превышает аналогичные показатели титана.
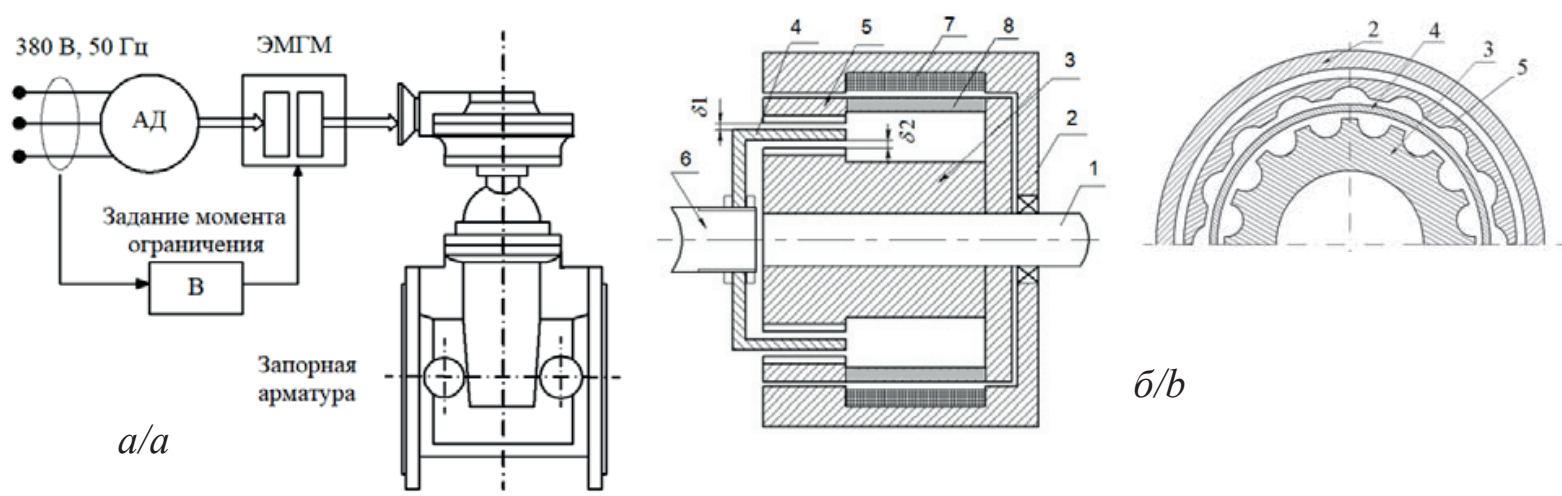

Pис. 1. Электропривод запорной арматуры с электролагнитной гистерезисной муфтой (а) и ее конструкция (б): 1 - ведущий вал; 2 наружный индуктор; 3 - внутренний индуктор; 4- гистерезисный слой; 5-внешний индуктор; 6-ведомый вал; 7 - неподвижная обмотка возбуждения; 8 - немагнитная вставка; $\delta 1, \delta 2$ - рабочие зазоры

Fig. 1. Valves electric drive with electromagnetic hysteresis clutch ( $a$ ) and its design ( $b$ ): 1 is the driving shaft; 2 is the external inductor; 3 is the internal inductor; 4 is the hysteresis layer; 5 is the outdoor inductor; 6 is the driven shaft, 7 is the field winding; 8 is the non-magnetic insert; $\delta 1, \delta 2$ are the working air gaps 
Теория гистерезисных муфт изложена, в частности, в работах $[7,8,13,14]$, на основе чего появился ряд отечественных изобретений, например [15-17]. Среди зарубежных патентов можно выделить работы [18-20]. Следует отметить, что в теоретических исследованиях указанных выше авторов практически не рассмотрены вопросы перемагничивания гистерезисных материалов в зависимости от конструктивных особенностей муфт, в частности от геометрии зубцовой зоны. Упрощения, принятые вышеуказанными авторами, объясняются математическими трудностями, связанными с учетом нелинейности зависимости $B=F(H)$, а также непостоянством магнитной проницаемости и гистерезисного угла. Кроме того, известно, что гистерезисные потери зависят не только от индукции в материале, но и от типа перемагничивания (линейное - тангенциальное или радиальное, и вращательное).

На рис. 2 представлен фрагмент зубцовой зоны ЭМГМ из магнитомягкого материала и гистерезисного слоя. Из рисунка следует, что в зависимости от относительного сдвига зубцов внутреннего и наружного индуктора точка «m», представляющая собой элементарный объем гистерезисного материала, в асинхронном режиме испытывает различные воздействия от изменяющегося магнитного поля. На рис. 2, а в элементарный объем «m» перемагничивается по полному циклу петли гистерезиса материала при тангенциальном поле по траектории $a-b-c-e-f-a$, а на рис. $2, \sigma, \varepsilon-$ по частному циклу петли при радиальном поле по траектории $a-b-c-d-a$, что в обоих случаях можно отнести к линейному перемагничиванию и пользоваться видом петли гистерезиса, получаемой при помощи «классических» установок по снятию петель [5, 9].
В общем случае, в зависимости от толщины гистерезисного слоя, величины воздушного зазора и геометрии зубцовой зоны, картина распределения магнитного потока может выглядеть сложнее, с элементами вращательного гистерезиса.

Решение задачи оптимизации зубцовой зоны ЭМГМ на основе моделирования в ANSYS MAXWELL

Получение различных аналитических зависимостей момента ЭМГМ практически невозможно, а задачу оптимизации можно решить с применением пакета ANSYS MAXWELL, успешно используемого при расчетах магнитных полей. В соответствии с трехмерной моделью ЭМГМ, сформированной в ANSYS MAXWELL (рис. $3, a$ ), на рис. 3 , б представлена картина распределения магнитного поля в поперечном разрезе зубцовой зоны муфты с неподвижной внешней обмоткой.

Результаты имитационного моделирования ЭМГМ показаны на рис. 4, 5. Как следует из рис. 3 , б, представляющего картину распределения магнитного поля в плоском разрезе ГМ, вектор магнитной индукции в зубцовой зоне совершает колебания около радиального направления. Радиальная составляющая магнитного поля практически не изменяется, поскольку суммарная величина воздушных зазоров при движении точки «m» остается постоянной. Такое перемагничивание материала можно характеризовать как частичное вращательное, в результате чего точка "m», представляющая элементарный объем активного слоя, совершает движение по симметричному циклу петли гистерезиса под воздействием тангенциальной составляющей поля.

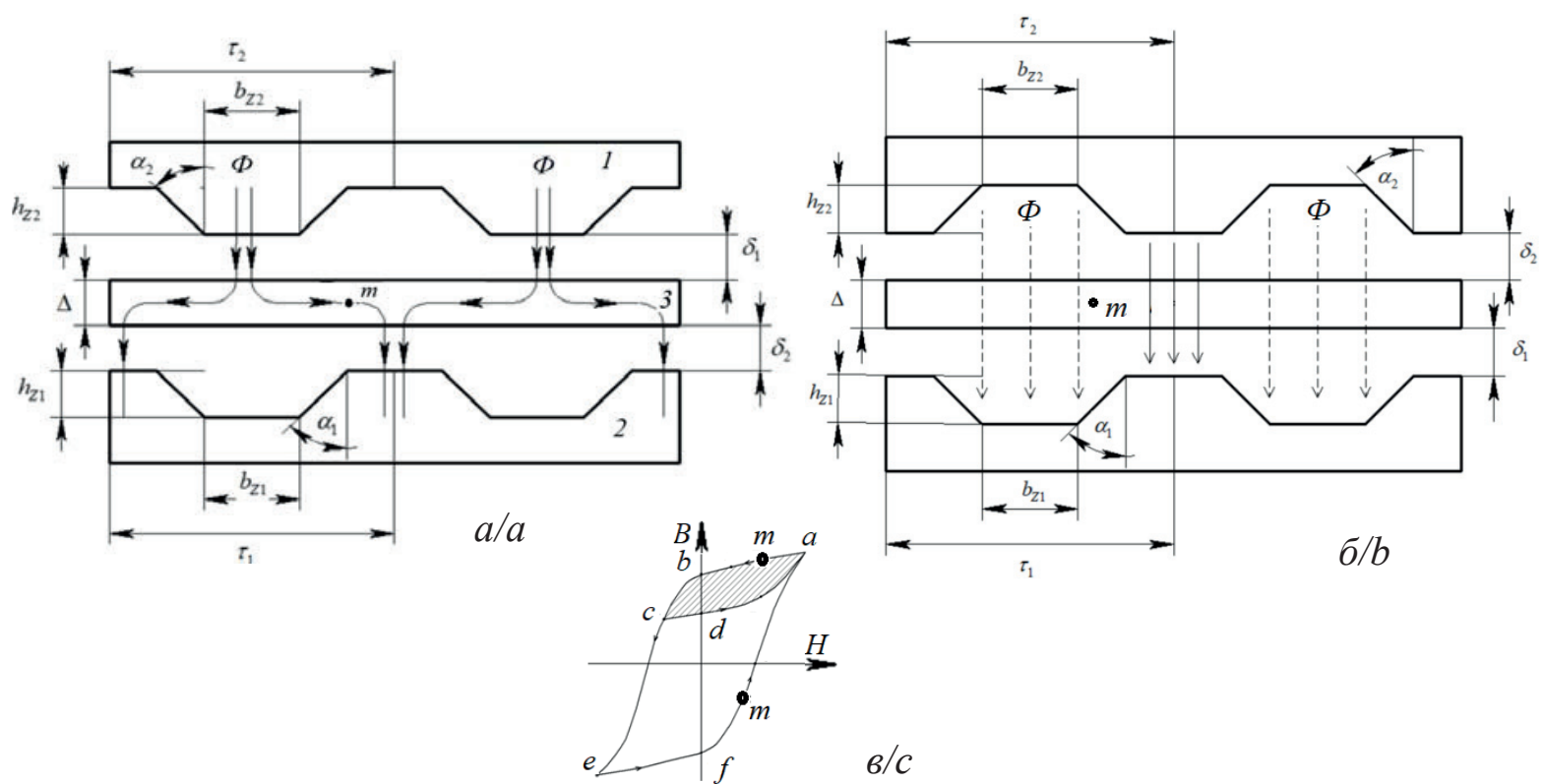

Pис. 2. Фраглент зубиовой зоны электромагнитной гистерезисной муфты: 1,2 - наружный и внутренний индуктор; 3 - гистерезисный слой; а-тангенциальное перемагничивание; б - радиальное перемагничивание; в - траектория точки «т»

Fig. 2. Piece of the electromagnetic hysteresis clutch teeth zone: 1, 2 are the external and internal inductors; 3 is the hysteresis layer: a is the tangential magnetization reversal; $b$ is the radial magnetization reversal; $c$ is the trajectory of the point «m» 

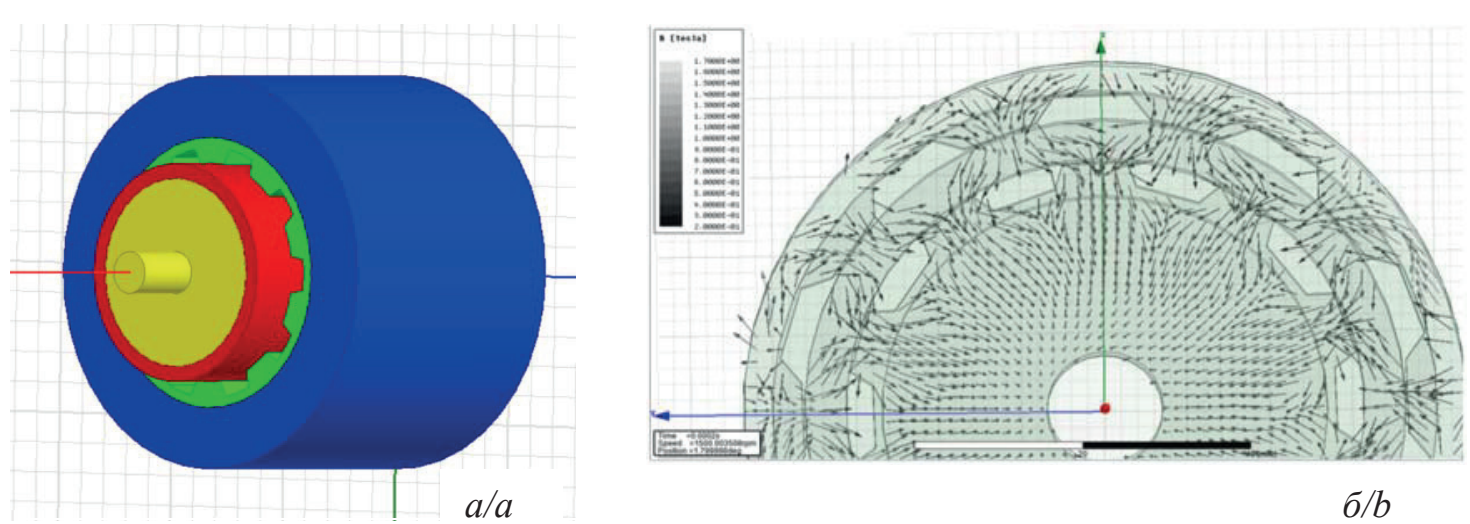

Рис. 3. Трехмерная модель электромагнитной гистерезисной муфты (а) и распределение магнитного поля в электролагнитной гистерезисной муфте (б)

Fig. 3. Electromagnetic hysteresis clutch three-dimensional model (a) and the magnetic field distribution in electromagnetic hysteresis clutch (b)

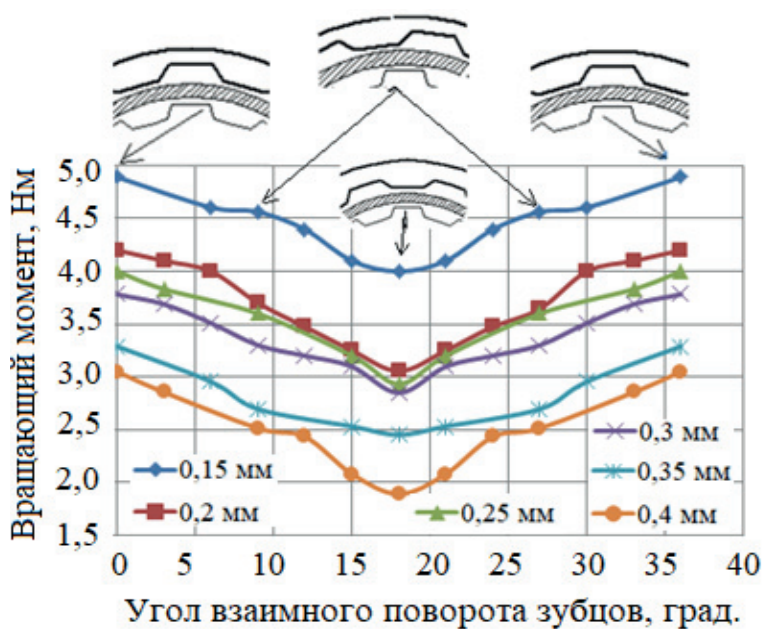

Pис.4. Зависилость вращающего момента электромагнитной гистерезисной муфты от взаимного расположения зубиовых зон индукторов и величины воздушного зазора

Fig. 4. Dependence of the electromagnetic hysteresis clutch torque on the relative position of the inductors teeth zones and the air gap size

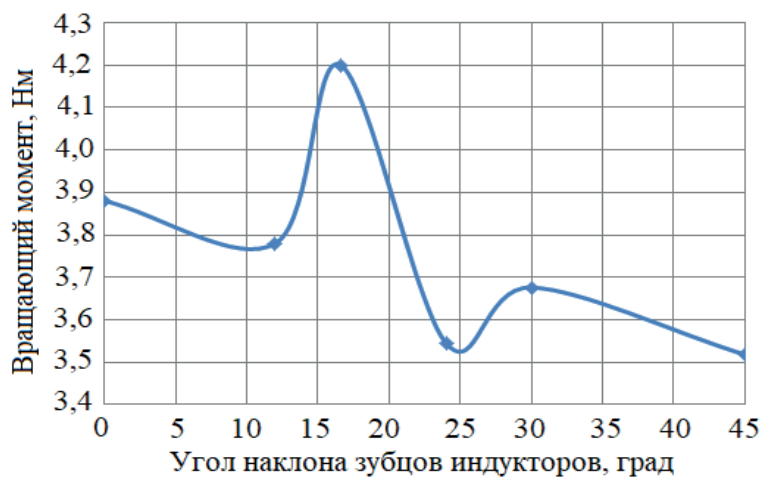

Pис.5. Зависилость вращающего момента электромагнитной гистерезисной муфты от угла наклона зубиов индукторов

Fig. 5. Dependence of the electromagnetic hysteresis clutch torque on the inductors teeth inclination angle

Из рис. 4 следует, что наибольший момент в ЭМГМ развивается при уменьшении воздушного зазора $\delta$ и расположении зубцов согласно рис. $3, \sigma$, что объясняется возрастанием тангенциальной со- ставляющей поля по мере сдвига зубцов внутреннего индуктора относительно наружного. Что касается конструкции зубцов, то, как следует из результатов моделирования, существует некоторое оптимальное значение угла $\alpha_{i}$ наклона зубцов индукторов, при котором развивается наибольший вращающий момент (рис. 5). Физическое обоснование этого факта состоит в следующем.

При постоянстве размеров воздушных зазоров $\delta_{i}$, толщины гистерезисного слоя $\Delta$, размерах $h$ и $r$ наклон зубцов сопровождается изменением ширины зубцов $b$ и угла наклона $\alpha$. При этом угол $\alpha$ изменяется от нуля до некоторого предельного значения $\alpha_{\mathrm{mp}}=\operatorname{arctg}(r / 2 h)$ при получении зубцов треугольной формы и $b=0$. При нулевом угле $\alpha$ зубцы имеют форму прямоугольника. При уменьшении ширины зубцов и увеличении угла $\alpha$ начинается снижение величины общего магнитного потока $\Phi$ ввиду возрастания магнитного сопротивления эквивалентного воздушного зазора, что ведет к снижению момента ЭМГМ. Увеличение угла $\alpha$ и уменьшение ширины зубцов $b$ приводит к возрастанию тангенциальной составляющей магнитного потока и резкому увеличению момента. При дальнейшем увеличении угла $\alpha$ на фоне снижения общего магнитного потока и его тангенциальной составляющей момент ЭМГМ снижается. Однако при уменьшении ширины зубцов происходит их локальное насыщение и, как следствие, некоторое увеличение тангенциальной составляющей индукции. Последующее увеличение угла до предельного значения $\alpha_{\text {пр }}$ вырождает зубцы в треугольные при снижении магнитного потока и момента муфты. Таким образом, в графике зависимости вращающего момента ЭМГМ от наклона зубцов внутреннего и внешнего индукторов наблюдаются два экстремума.

При варьировании параметров зубцов лишь внешнего индуктора и постоянных значениях наклона и ширины зубцов внутреннего индуктора зависимость момента от угла наклона аналогична рис. 5 (рис. 6) при некоторых количественных отличиях. В эксперименте также фиксировалось значение тангенциальной составляющей индук- 

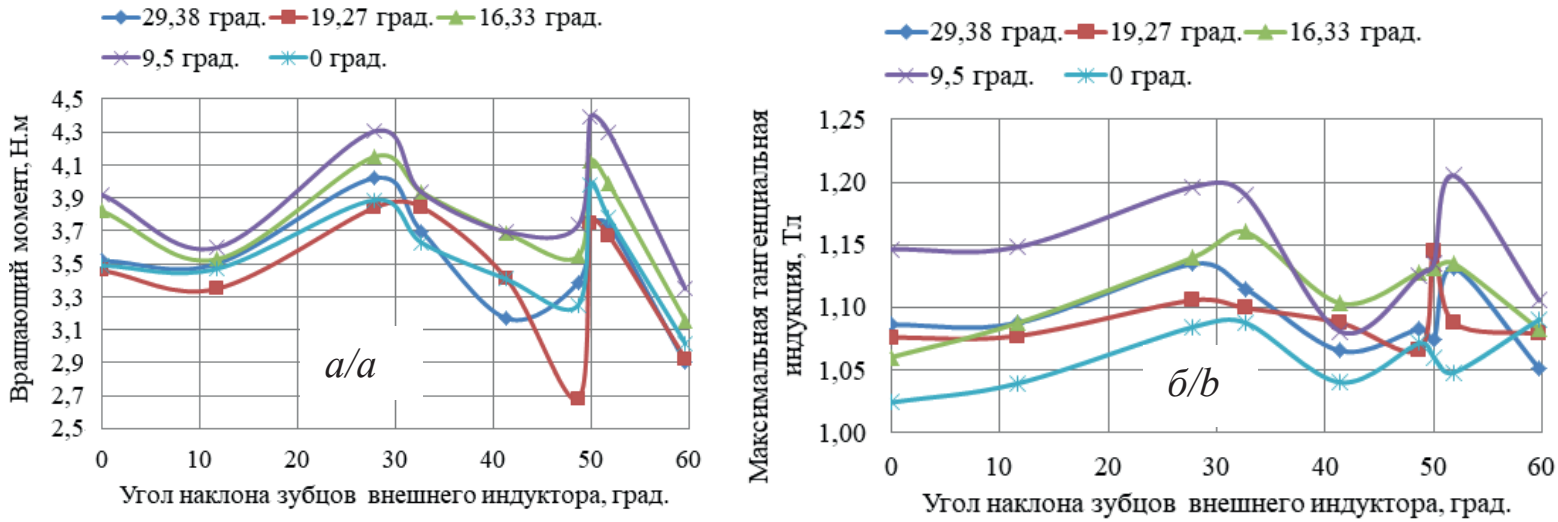

Pис. 6. Зависилости вращающего молента (а) и максилальной тангенциальной индукиии (б) от угла наклона зубиов внешнего индуктора при разных углах наклона зубцов внутреннего индуктора при числе зубцов $z=10$

Fig. 6. Dependences of the torque (a) and maximum tangential magnetic flux density (b) on the external inductor teeth inclination angle at different inclination angles of the internal inductor teeth when the teeth number is $z=10$

ции в гистерезисном слое (рис. 6, б). Как следует из рис. 6 , варьирование шириной зубцов индукторов приводит к аналогичным зависимостям.

Как было отмечено выше, электромагнитный момент и эффективность ЭМГМ определяются тан- генциальной составляющей магнитного потока в гистерезисном слое. Для оценки указанной эффективности введем коэффициент использования гистерезисного слоя по тангенциальной составляющей магнитного поля

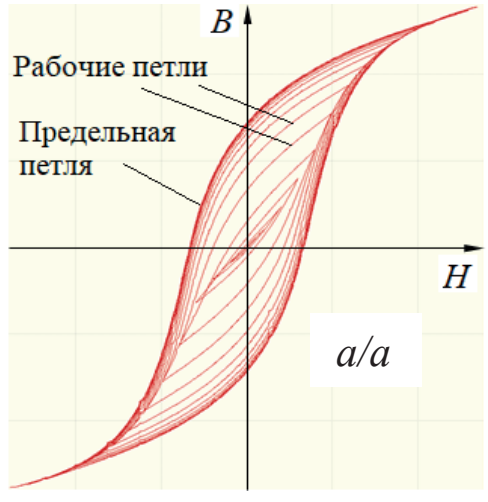

$\rightarrow 6 \mathrm{MM} \leftrightarrows-7 \mathrm{MM} \leftarrow 7,5 \mathrm{MM} \leftarrow 7,8 \mathrm{MM} \leftarrow 8,5 \mathrm{MM}$

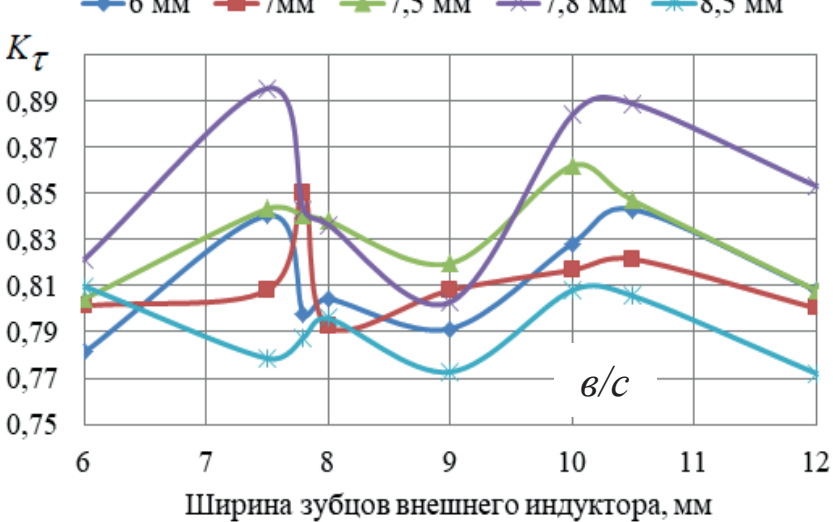

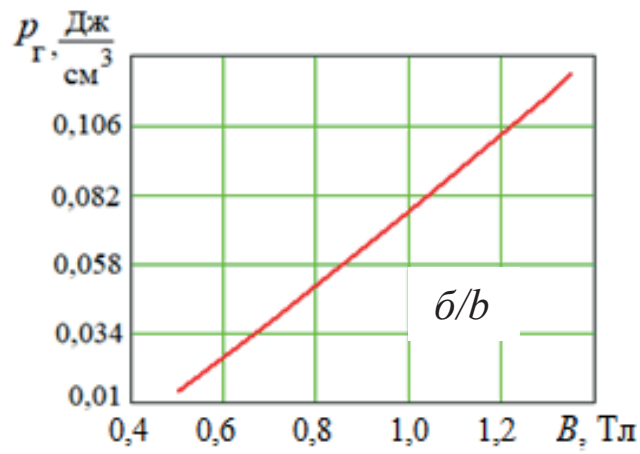

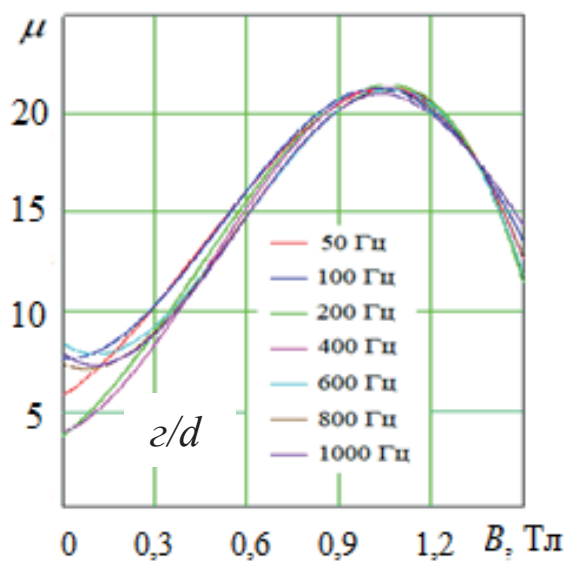

Pис. 7. Семейство петель гистерезиса (а), удельные гистерезисные потери (б), кривые коэффициента использования гистерезисного слоя по тангенциальной составляющей индукции в зависимости от ширины зубиов (в) и относительная магнитнал проницаемость (г) в функиии от индукиии для материала $22 X 15 K A$ (кривая $\mu=F$ (B) дана при различных частотах перемагничивания гистерезисного слоя)

Fig. 7. Family hysteresis loops (a), the specific hysteresis losses (b), the curve of the hysteresis layer efficiency in relation to the magnetic flux density tangential component depending on the teeth width $(c)$ and the relative magnetic permeability $(d)$ as functions on the magnetic flux density for 22X15KA material (the curve $\mu=F(B)$ is given at different hysteresis layer magnetization frequencies) 


$$
K_{\tau}=\frac{p_{\text {pa }}}{p_{\text {max }}}=\frac{\oint H_{\text {pa } \tilde{}} d B}{\oint H_{\text {max }} d B},
$$

где $p_{\max }, p_{\text {раб }}$ и $H_{\text {max }}, H_{\text {раб }}$ - удельные потери на гистерезис и напряженность поля при максимальной и рабочей индукции соответственно.

Тогда выражение для момента ЭМГМ при объеме гистерезисного слоя $V_{\text {г }}$ пимет вид:

$$
M_{\mathrm{r}}=p_{\mathrm{r}} V_{\mathrm{r}} K_{\tau} \text {. }
$$

На рис. 7, а представлено семейство петель гистерезиса материала Fe-Cr-Co (сплав 22X15KA), полученное авторами на установке контроля магнитных параметров УКМП-0,05-100, на основе чего были построены зависимости удельных гистере-

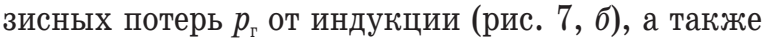
магнитной проницаемости гистерезисного материала от индукции и частоты перемагничивания (рис. 7, 2). Фактически круговые интегралы в выражении (1) представляют из себя площади петель гистерезиса в соответствии с рис. 7, a. При этом тангенциальная составляющая индукции $B_{\tau}$ может быть представлена как

$$
B_{\tau}=\mu_{0} \mu H_{\tau},
$$

где $\mu_{0}$ и $\mu$ - соответственно магнитная проницаемость вакуума и относительная магнитная проницаемость гистерезисного материала.

На рис. 7, в представлены зависимости коэффициента использования гистерезисного слоя по тангенциальной составляющей от ширины зубцов, коррелирующие с рис. 6.
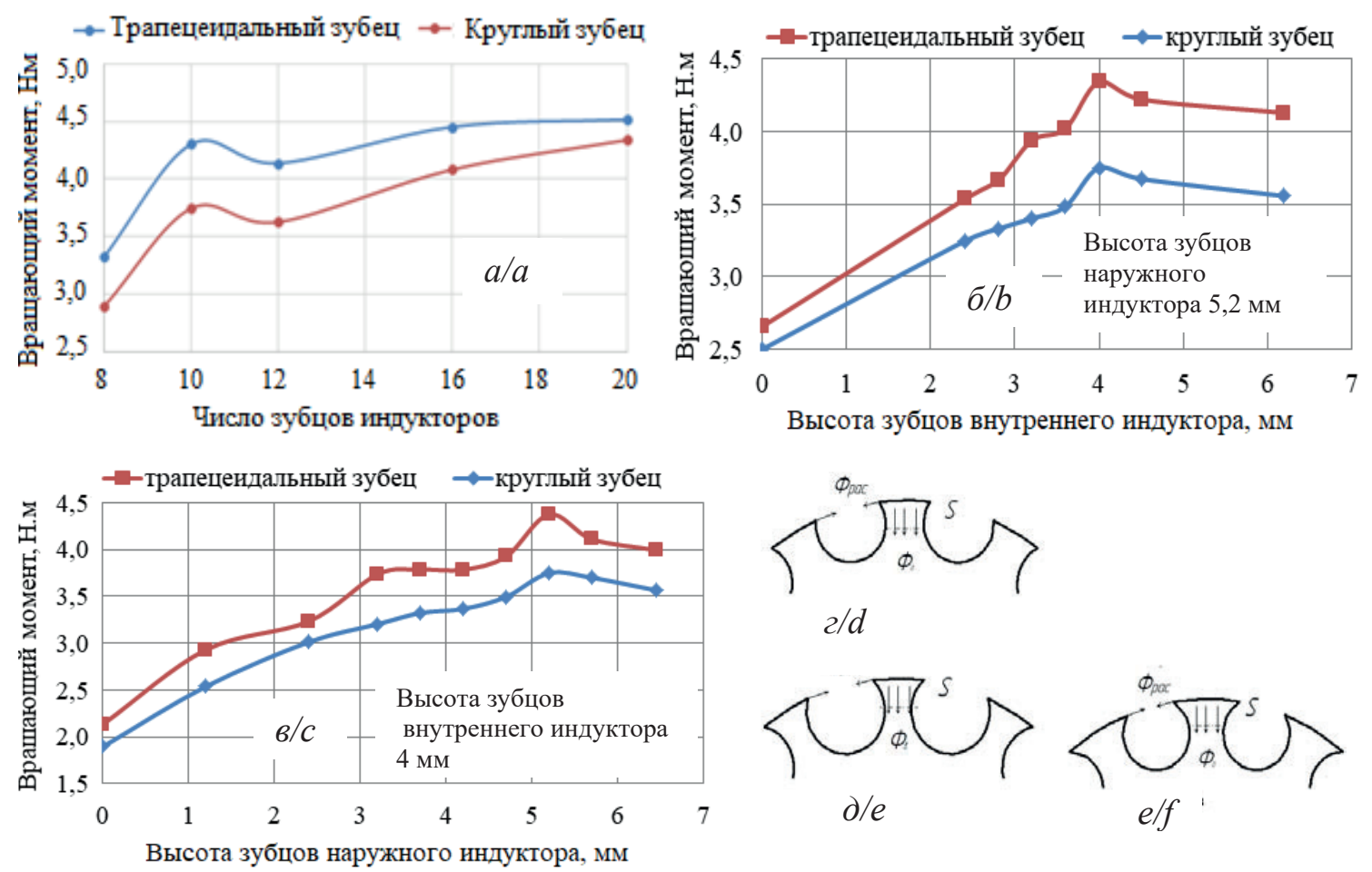

Проведенные исследования позволяют более глубоко и наглядно представить процессы перемагничивания гистерезисного слоя в составе ЭМГМ, что, в свою очередь, приводит к возможности оптимизации электромагнитной системы муфты с целью получения максимального вращающе-
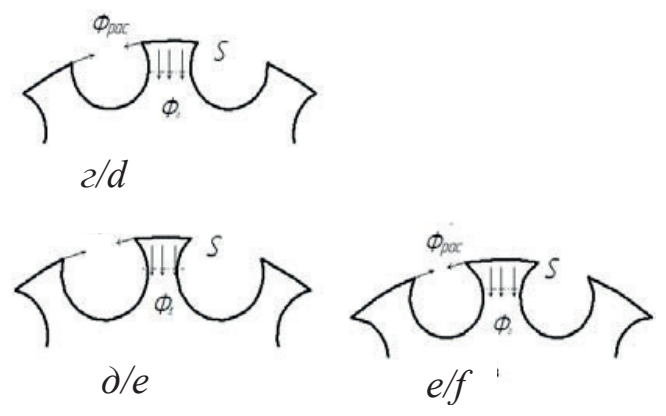

Pис. 8. Зависимость вращающего момента электролагнитной гистерезисной муфты от числа зубцов индукторов при различных формах пазов (а), размеров зубиов индукторов $(б$, в) и фраглент зубиовой зоны с круглыми пазами (г-е)

Fig. 8. Dependence of the electromagnetic hysteresis clutch torque on the teeth inductors number for various slots forms (a), inductors teeth dimensions $(b, c)$, and a piece of the teeth zone with round slots $(d-f)$. 

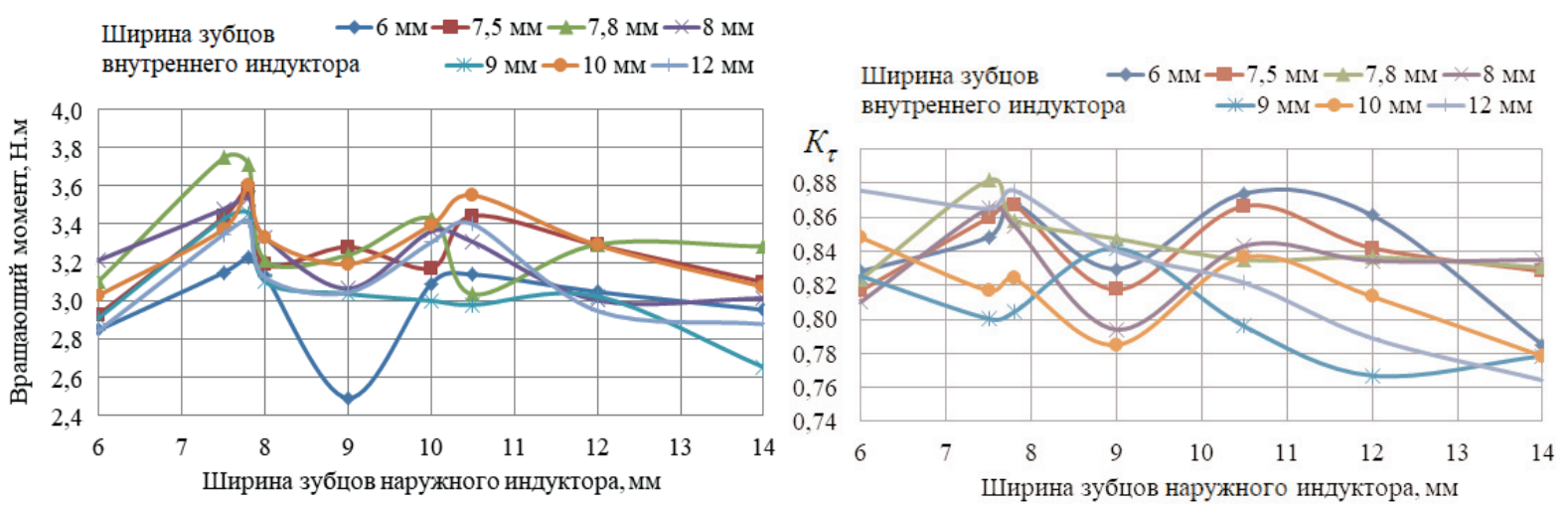

Рис.9. Зависимость момента и коэффициента использования гистерезисного слоя от ширины зубиов наружного индуктора при разных ширинах зубиов внутреннего индуктора для круглых зубиов при $z=10$

Fig. 9. Dependence of the torque and the hysteresis layer efficiency on the external inductor teeth width at different widths of the internal inductor teeth for round teeth when the teeth number is $z=10$

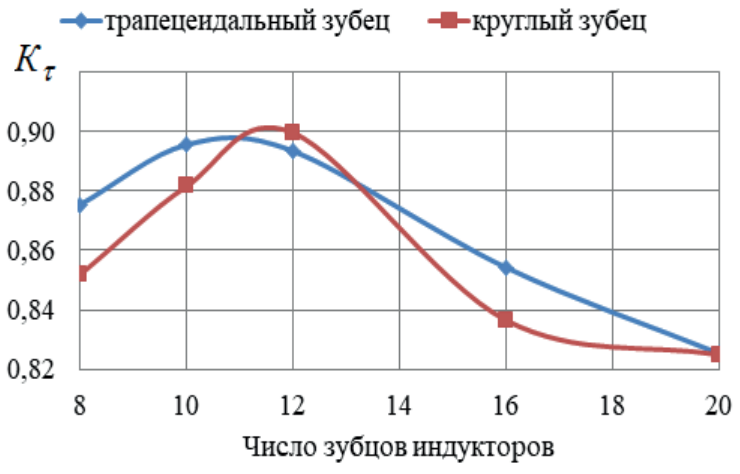

Pис. 10. Зависилость коэффициента использования гистерезисного слоя по тангенииальной составляющей от числа зубиов индукторов

Fig. 10. Dependence of the hysteresis layer efficiency in relation to the magnetic flux density tangential component depending on the teeth inductors number

го момента при фиксированных габаритах и массе. Результаты исследования, представленные в настоящей статье, а также в работе [21], показывают, что свойства сплава типа $22 \mathrm{X} 15 \mathrm{KA}$ на основе $\mathrm{Fe}$ $\mathrm{Cr}-\mathrm{Co}$ в части магнитной проницаемости слабо зависят от частот перемагничивания вплоть до частоты 2000 Гц, что при его высокой прочности по-

\section{СПИСОК ЛИТЕРАТУРЫ}

1. Гарганеев А.Г., Каракулов А.С., Ланграф С.В. Электропривод запорной арматуры. - Томск: Томский политехнический университет, 2013. -157 с.

2. Гуревич Д.Ф. Трубопроводная арматура: справочное пособие. 2-е изд., переработанное и дополненное. - Л.: Машиностроение, Ленинградское отделение, 1981. - 368 с.

3. Васин С.А., Плахотникова Е.В. Методика расчета величины крутящего момента настройки электропривода в системе электроприводной запорной арматуры с прямолинейным перемещением запорного органа // Записки Горного института. 2018. - T. 232. - C. 407-412.

4. Гарганеев А.Г., Каракулов А.С., Ланграф С.В. Электропривод запорной арматуры как мехатронная система // Научный вест- зволяет конструировать ЭМГМ на высокие окружные скорости. Зубцовая зона ЭМГМ с зубцами трапецеидальной формы дает лучшее использование геометрических размеров муфты для достижения максимального момента по сравнению с зубцами круглой формы. Оптимальным значением углов наклона зубцов следует считать значения $\alpha=9,5^{\circ}$ для внутреннего индуктора и $\alpha=27,8^{\circ} ; \alpha=57,8^{\circ}$ для наружного индуктора при относительном сдвиге зубцов индукторов наполовину зубцового шага.

\section{Заключение}

Результаты моделирования подтверждают и дополняют расчетные данные, полученные ранее авторами, в частности, в работах $[5,9]$. Таким образом, вариант ЭПЗА с ЭМГМ и АД благодаря простоте его реализации, возможности регулирования и ограничения момента в широком диапазоне температур и окружных скоростей можно рассматривать как перспективную альтернативу ЭП с частотным управлением и тиристорными регуляторами. Следует отметить, что, в принципе, положительные особенности гистерезисных муфт удачно сочетаются со спецификой работы технологических механизмов различных устройств, что и объясняет интерес разработчиков к такого рода электромеханическим преобразователям [22-30].

ник Новосибирского государственного технического университета. - 2013. - № 2. - С. 180-187.

5. Гарганеев А.Г., Кюи Д.К., Кашин Е.И. Электропривод задвижки трубопроводной арматуры с гистерезисной муфтой // Доклады Томского университета систем управления и радиоэлектроники. - 2018. - Т. 21. - № 1. - С. 127-131.

6. Ануфриев В.П., Чвялев Д.С., Волин А.О. Запорная и регулирующая арматура. Многократное увеличение срока службы // Арматуростроение. - 2018. - № 5 (116). - С. 49-51.

7. Мизюрин С.Р., Ермилов М.А. Проектирование магнитно-гистерезисных муфт. - М.: Ротапринт, Московский авиационной институт, 1966. - 123 с.

8. Ганзбург Л.Б., Федотов А.И. Проектирование электромагнитных и магнитных механизмов: справочник. - Л.: Машиностроение, Ленинградское отделение, 1980. - 364 с. 
9. Гистерезисные муфты на основе материала Fe-Cr-Co / А.Г. Гарганеев, Д.К. Кюи, Е.И. Кашин, Н.Ю. Сипайлова // Горное оборудование и электромеханика. - 2018. -№ 4 (138). - С. 33-38.

10. Практика и дальнейшие перспективы промышленного использования высокопрочных наноструктурных магнитотвердых сплавов системы Fe-Cr-Co // Г.С. Бурханов, А.Г. Дормидонтов, И.М. Миляев, В.С. Юсупов // Нанотехнологическое общество России. URL: http: www.ntsr.info/science/library/3201.htm (дата обращения: 15.05.2019).

11. Магнитные гистерезисные и механические свойства магнитотвёрдого сплава 27Х15К2МСТФ / И.М. Миляев, В.С. Юсупов, С.Ю. Останин, С.И. Стельмашок, А.И. Миляев, Н.В. Лайшева / Физика и химия обработки материалов. - 2017. - № 1. C. $69-76$.

12. Вомпе Т.А. Разработка и исследование низкокобальтовых магнитотвердых Fe-Cr-Co-сплавов: дис. ... канд. техн. наук. - М., 2018. -155 c.

13. Мастяев Н.З., Орлов И.Н. Гистерезисные электродвигатели. Ч. I. - М.: Московский энергетический институт, 1963. $220 \mathrm{c.}$

14. Тарасов В.Н., Делекторский Б.А. Управляемый гистерезисный привод. - М.: Энергоатомиздат, 1983. - 128 с.

15. Управляемая гистерезисная муфта: Авторское свидетельство СССР № 604098, Н02К 49/04. - Бюл. № 15 от 25.04.78. - 3 с.

16. Управляемая гистерезисная муфта: Авторское свидетельство СССР № 332552, Н02К 49/04. - Бюл. № 10 от 19.04.72. - 2 с.

17. Электромагнитная гистерезисная муфта: Авторское свидетельство СССР № 442556, Н02К 49/04. - Бюл. № 33 от 05.09.74. $-2 \mathrm{c}$.

18. Hysteresis Clutch for Film Winding: patent № US3934833, Jan. $27,1976$.

19. Hysteresis clutch: patent № US2912607A, July 07, 1956.

20. Magnetic hysteresis clutch: patent № US5581139A, August 25, 1993.

21. Гарганеев А.Г., Кашин Е.И., Кашеутов А.В. 0 фильтрующих свойствах электромагнитной системы гистерезисных гиродвигателей в мехатронных устройствах с использованием инфор- мативных свойств автономного инвертора // Электричество. 2019. - № 1. - C. 55-66.

22. Zhou Wu. Comprehensive Calculating Method of Friction Moment for Circle Disc Frictional Cultch / Journal of Shaanxi Institute of Technology. - 2004. - V. 20. - № 4. - P. 4-11.

23. Kielan P., Kowol P., Pilch Z. Conception of the electronic controlled magneto-rheological clutch // Electrical Review. - 2011. V. 87. - № 3. - P. 93-95.

24. Yuliang S., Shaopu Y., Cunzhi P. Experimental research of magneto-rheological fluid clutch / IEEE International Conference on Vehicular Electronics and Safety. - 2005. - P. 104-107.

25. Magnetic Properties of Hard Magnetic Alloy of Fe-27Cr-10Co2Mo / M.I. Alymov, I.M. Milyaev, V.S. Yusupov, V.A. Zelensky, A.I. Milyaev, A.B. Ankudinov, D.M. Abashev // Proc. of the $14^{\text {th }}$ Sino-Russia Symposium on Advanced Materials and Technologies by the Nonferrous Metals Society of China. - 2018. - P. 64-68. D0I: $10.1088 / 1757-899 \mathrm{X} / 347 / 1 / 012053$.

26. Shock-resistance rotor design of a high-speed PMSM for integrated pulsed power system Wan Yuan, Cui Shumei, Wu Shaopeng, Song Liwei, I.M. Milyaev, S.Yu. Ostanin / IEEE Transactions on Plasma Science. - 2017. - V. 45. - № 7. - P. 1399-1405.

27. Analysis of a modular pulsed alternate power system for driving 32-MJ muzzle energy railgun / Cui Shumei, Wang Shaofei, Wu Shaopeng, S.Yu. Ostanin, I.M. Milyaev // IEEE Transactions on Plasma Science. - 2017. - V. 45. - № 7. - P. 1128-1133.

28. Proposal of electromagnetic clutch structure to suppress surge voltage / J. Muramatsu, T. Kojima, H. Tanaka, Y. Hattori, H. Okada, H. Keino, T. Nakanishi, K. Fujisaki // IEEE Transactions on Magnetics. - 2014. - V. 50. - № 11. - P. 1-4.

29. Wenjun Li. Design and Development of Magneto-Rheological Actuators with Application in Mobile Robotics: Thesis and Dissertation Repository. - Western Ontario, 2014. - 95 p.

30. Shafer A.S., Kermani M.R. On the feasibility and suitability of $\mathrm{mr}$ fluid clutches in human-friendly manipulators // Mechatronics, IEEE/ASME Transactions. - 2011. - № 99. - P. 1-10.

Поступила 31.05.2019 2.

\section{Информация об авторах}

Гарганеев А.Г., профессор, доктор технических наук, профессор Инженерной школы энергетики Национального исследовательского Томского политехнического университета.

Динь $\boldsymbol{\kappa} . \boldsymbol{\kappa}$., аспирант инженерной школы энергетики Национального исследовательского Томского политехнического университета.

Сипайлова H.Ю., кандидат технических наук, доцент Инженерной школы энергетики Национального исследовательского Томского политехнического университета.

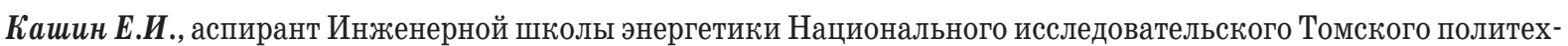
нического университета. 


\title{
OPTIMIZATION OF HYSTERESIS CLUTCH TEETH ZONE GEOMETRY IN OIL PIPELINE SHUT-OFF VALVES
}

\author{
Aleksandr G. Garganeev', \\ garganeev@rambler.ru \\ Din K. Kyui', \\ caothuhanam89@gmail.com
}

\section{Nadezhda Yu. Sipaylova',}

snu2002@mail.ru

\author{
Evgeniy I. Kashin', \\ kashinei@sibmail.com \\ 1 National Research Tomsk Polytechnic University, \\ 30, Lenin Avenue, Tomsk, 634050, Russia.
}

The relevance of the paper is related to the search for new efficient technical solutions in the sphere of electric drives for oil pipeline valves based on using the hysteresis principle of electromechanical energy conversion and new hard magnetic materials.

The main aim of the research is to reveal with the analysis of the hysteresis layer magnetization the teeth zone optimal geometry of the electromagnetic hysteresis clutch based on the $\mathrm{Fe}-\mathrm{Cr}$-Co alloy to obtain the maximum torque.

The methods: experimental methods for studying the magnetic characteristics of magnetically hard materials using the original laboratory installations; simulation in the ANSYS MAXWELL, the theoretical methods of electromagnetic field analysis.

The results. The authors have obtained and investigated torque dependences on the teeth zone geometric parameters for the clutches based on Fe-Cr-Co 22X15KA alloy. To value the hysteresis material efficiency, the authors introduced the hysteresis layer use coefficient according to the magnetic field tangential component. The model of the electromagnetic hysteresis clutch with fixed control winding, taking into account the parameters of the alloy of Fe-Cr-Co 22X15KA was developed in the ANSYS MAXWELL program. To obtain the maximum torque at the limited clutch sizes and mass the optimal teeth zone geometric dimensions were determined. It is concluded, that the pipeline shut-off valves electric drive with hysteresis clutch and induction motor due to the ease of implementation, the possibility of regulating and limiting the torque in the wide temperatures and rotation speeds range, can be considered as a promising alternative to electric drives with frequency control and thyristor regulators.

\section{Key words:}

Electromagnetic clutch, simulation, electrical drive, torque, hysteresis, hard magnetic material, valves, oil pipeline.

\section{REFERENCES}

1. Garganeyev A.G., Karakulov A.S., Langraf S.V. Elektroprivod zapornoy armatury [The shutoff valves of electric drive]. Tomsk, Tomsk Polytechnic University Publ., 2013. 157 p.

2. Gurevich D.F. Truboprovoadnya armatura: spravochnoe posobie [The pipelines shutoff valves: handbook]. Leningrad, Mashinostroyeniye Publ., 1981. 368 p.

3. Vasin S.A., Plakhotnikova S.A. The calculation method of the electric drive torque setting in the electrically-driven shutoff valves with linear movement of the locking element. Zapiski Gornogo instituta, 2018, no. 232, pp. 407-412. In Rus.

4. Garganeyev A.G., Karakulov A.S., Langraf S.VThe shutoff valves electric drive as mechatronic system. Scientific Bulletin of Novosibirsk State Technical University, 2013, no. 2, pp. 180-187. In Rus.

5. Garganeev A.G., Kyui D. K., Kashin E. I. The electric drive with hysteresis clutch for pipeline valves. Proceedings of Tomsk State University of Control Systems and Radioelectronics, 2018, vol. 21, no. 1, pp. 127-131. In Rus.

6. Anufriev V.P., Chvyalev D.S., Volin A.0. The shutoff and control valves. Repeated increase of the service life. Armaturostroenie, 2018, no. 5 (116), pp. 49-51. In Rus.

7. Mizyurin S.R., Ermilov M.A. Proektirovanie magnitno-gisterezisnoy mufty [The magnetic hysteresis clutch development]. Moscow, Moscow Aviation Institute Publ., 1966. 123 p.

8. Ganzburg L.B., Fedotov A.I. Proektirovanie elektromagnitnykh $i$ magnitnykh mekhanizmov: spravochnik. [The electromagnetic and magnetic mechanisms development: Handbook]. Leningrad, Mashinostroyenie Publ., 1980. 364 p.
9. Garganeev A.G., Kyui D.K., Kashin E.I., Sipajlova N.Yu. Hysteresis clutches based on the Fe-Cr-Co material. Mining equipment and electromechanics, 2018, no. 4 (138), pp. 33-38. In Rus.

10. Burhanov G.S., Dormidontov A.G., Milyaev I.M., Yusupov V.S. Praktika i dalneyshie perspektivy promyshlennogo ispolzovaniya vysokoprochnykh nanostrukturnykh magnitotverdykh splavov sistemy $\mathrm{Fe}-\mathrm{Cr}-\mathrm{Co}_{0}$ [Practice and future prospects industrial use of high-strength nanostructured hard magnetic alloys of $\mathrm{Fe}-\mathrm{Cr}-\mathrm{Co}]$. Nanotekhnologicheskoe obshchestvo Rossii. Available at: http: www.ntsr.info/science/library/3201.htm (accessed 15 May 2019).

11. Milyaev I.M., Yusupov V.S., Ostanin S.Yu., Stelmashok S.I., Milyaev A.I., Laysheva N.V. Magnetic hysteresis and mechanical properties of hard magnetic alloy of $27 \mathrm{Kh} 15 \mathrm{~K} 2 \mathrm{MSTF}$. Physics and chemistry of materials treatment, 2017, no. 1, pp. 69-76. In Rus.

12. Vompe T.A. Razrabotka i issledovanie nizkokobaltovykh magnitotverdykh Fe-Cr-Co splavov. Dis. Kand. nauk [Development and research of low cobalt hard magnetic alloy of $\mathrm{Fe}-\mathrm{Cr}-\mathrm{Co}$. Cand. Diss.]. Moscow, 2018. $155 \mathrm{p}$.

13. Mastyaev N.Z., Orlov I.N. Gisterezisnye elektrodvigateli [Hysteresis electrical motors]. Moscow, Moscow Power Engineering Institute Publ., 1963. 220 p.

14. Delektorskiy B.A., Tarasov V.N. Upravlyaemy gisterezisny privod [Hysteresis controlled drive]. Moscow, Energoatomizdat Publ., 1983. 128 p.

15. Yakovlev V.Ya. Upravlyaemaya gisterezisnaya mufta [Hysteresis controlled clutch]. Patent no. 604098, 1978.

16. Gordeev N.V. Upravlyaemaya gisterezisnaya mufta [Hysteresis controlled clutch]. Patent no. 332552, 1972. 
17. Kovalev N.P., Kharlamov N.V., Korsmik A.A. Elektromagnitnaya gisterezisnaya mufta [Electromagnetic hysteresis clutch]. $\mathrm{Pa}$ tent no. 442556, 1974.

18. Nash J.L., Falls G., Carrico Ph.H. Hysteresis Clutch for Film Winding. Patent no. US3934833, 1976.

19. Duncan J.E. Hysteresis clutch. Patent no. US2912607A, 1956

20. Toukola R. Magnetic hysteresis clutch. Patent no. US5581139A, 1993.

21. Garganeev A.G., Kashin E.I., Kasheutov A.V. The filtering properties of the electromagnetic system of hysteresis hydraulic motors in mechatronic devices using the informative properties of the autonomous inverter. Elektrichestvo, 2019, no. 1, pp. 55-66. In Rus.

22. Zhou Wu. Comprehensive Calculating Method of Friction Moment for Circle Disc Frictional Cultch. Journal of Shaanxi Institute of Technology, 2004, vol. 20, no. 4, pp. 4-11.

23. Kielan P., Kowol P., Pilch Z. Conception of the electronic controlled magneto-rheological clutch. Electrical Review, 2011, vol. 87, no. 3, pp. 93-95.

24. Yuliang S., Shaopu Y., Cunzhi P. Experimental research of magneto-rheological fluid clutch. IEEE International Conference on Vehicular Electronics and Safety, 2005, pp. 104-107.

25. Alymov M.I., Milyaev I.M., Yusupov V.S., Zelensky V.A., Milyaev A.I., Ankudinov A.B., Abashev D.M. Magnetic Properties of
Hard Magnetic Alloy of Fe-27Cr-10Co-2Mo. Proc. of the $14^{\text {th }}$ SinoRussia Symposium on Advanced Materials and Technologies by the Nonferrous Metals Society of China, 2018, pp. 64-68. D0I: 10.1088/1757-899X/347/1/012053.

26. Wan Yuan, Cui Shumei, Wu Shaopeng, Song Liwei, Milyaev I.M., Ostanin S.Yu. Shock-resistance rotor design of a high-speed PMSM for integrated pulsed power system. IEEE Transactions on Plasma Science, 2017, vol. 45, no. 7, pp.1399-1405.

27. Cui Shumei, Wang Shaofei, Wu Shaopeng, Ostanin S.Yu., Milyaev I.M. Analysis of a modular pulsed alternate power system for driving 32-MJ muzzle energy railgun. IEEE Transactions on Plasma Science, 2017, vol. 45, no. 7, pp. 1128-1133.

28. Muramatsu J., Kojima T., Tanaka H., Hattori Y., Okada H., Keino H., Nakanishi T., Fujisaki K. Proposal of electromagnetic clutch structure to suppress surge voltage. IEEE Transactions on Magnetics, 2014, vol. 50, no. 11, pp. 1-4.

29. Wenjun Li. Design and Development of Magneto-Rheological Actuators with Application in Mobile Robotics: Thesis and Dissertation Repository. Western Ontario, 2014.95 p.

30. Shafer A.S., Kermani M.R. On the feasibility and suitability of MR fluid clutches in human-friendly manipulators. Mechatronics, IEEE/ASME Transactions on, 2011, no. 99, pp. 1-10.

Received: 31 May 2019.

\section{Information about the authors}

Aleksandr G. Garganeev, Dr. Sc., professor, National Research Tomsk Polytechnic University.

Din K. Kyui, postgraduate student, National Research Tomsk Polytechnic University.

Nadezhda Yu. Sipaylova, Cand. Sc., associate professor, National Research Tomsk Polytechnic University.

Evgeniy I. Kashin, postgraduate student, National Research Tomsk Polytechnic University. 